\title{
New Technologies for the Detection of Millimeter and Submillimeter Waves
}

\author{
P.L. Richards, ${ }^{1,2,4}$ J. Clarke, ${ }^{1,2}$ J.M. Gildemeister, ${ }^{1,2}$ T. Lanting, ${ }^{4}$ A.T. Lee, ${ }^{1,3}$ \\ M.J. Myers, ${ }^{1,4}$ D. Schwan, ${ }^{4}$ J.T. Skidmore, ${ }^{1,4}$ H.G. Spieler, ${ }^{3}$ \\ and Jongsoo Yoon ${ }^{1,2}$ \\ ${ }^{1}$ Physics Department, University of California, Berkeley, CA 94720-7300, U.S.A. \\ ${ }^{2}$ Materials Sciences Division, Lawrence Berkeley National Laboratory, Berkeley, CA 94720, U.S.A. \\ ${ }^{3}$ Physics Division, Lawrence Berkeley National Laboratory, Berkeley, CA 94720, U.S.A. \\ ${ }^{4}$ Space Sciences Laboratory, University of California, Berkeley, CA 94720-7450, U.S.A.
}

\begin{abstract}
Voltage-biased superconducting bolometers have many operational advantages over conventional bolometer technology including sensitivity, linearity, speed, and immunity from environmental disturbance. A review is given of the Berkeley program for developing this new technology. Developments include fully lithographed individual bolometers in the spiderweb configuration, arrays of 1024 close-packed absorber-coupled bolometers, antenna-coupled bolometers, and a frequency-domain SQUID readout multiplexer.
\end{abstract}

\section{INTRODUCTION}

The current generation of $\mathrm{CMB}$ experiments are done with two very different types of receiver. High electron mobility transistor (HEMT) amplifiers are phase-conserving linear photon amplifiers whose sensitivity is limited by quantum noise. Bolometers, by contrast, are square-law detectors which do not preserve phase so have no such sensitivity limit. In principle, bolometers can reach the sensitivity limit set by photon statistics. The best photometers using current spider web bolometers, with germanium thermistors and JFET amplifiers operate close to this limit for the photon rates observed near the peak of the CMB spectrum. In spite of their limited sensitivity, however, HEMT receivers are used for many current CMB temperature anisotropy experiments including the interferometers CBI, DASI, and VSI, and the MAP and PLANCK-LFI orbital missions. Part of the reasons for this preference is that the operating characteristics of HEMT amplifiers are generally superior to those of conventional bolometers. These characteristics include dynamic range, linearity, dependence of the responsivity on cryostat temperature and infrared power loading, speed, sensitivity to rf interference, required operating temperature, reproducibility, etc. These operating limitations make bolometric systems very difficult to optimize and often limit the performance achieved. Both HEMT and bolometer technologies are currently limited by complexity to relatively small arrays. The CMB interferometers have up to 14 receivers, bolometric systems use $16-100$ bolometers.

A number of cosmological experiments now under consideration will require much deeper or more rapid mapping of the sky than is now possible. These include proposed measurements of the $\mathrm{E}$ and $\mathrm{B}$-mode polarization anisotropy of the CMB, statistical studies of the Sunyaev-Zel'dovich effect and studies of the sources of the far infrared background. For these experiments we need large arrays of $10^{3}-10^{4}$ bolometers with improved operating characteristics.

\section{THE VOLTAGE-BIASED SUPERCONDUCTING BOLOMETER}

A new bolometric technology is now being developed which promises to supply the required performance in large format arrays. The voltage-biased superconducting bolometer (VSB) $[1,2]$ uses a superconducting thin film transition-edge temperature sensor (TES). The voltage bias creates a strong negative electrothermal feedback that keeps the bolometer operating temperature constant despite changes in cryostat temperature or 
infrared power loading. The VSB has a number of advantages over conventional bolometer technology. The feedback increases the bolometer speed and the linearity, and reduces the sensitivity to environmental factors. The Johnson noise is suppressed. The bolometers operate at low impedance, where microphonic effects are reduced, and use superconducting quantum interference (SQUID) amplifiers. These amplifiers dissipate negligible power, operate at low temperatures and have very low noise. Perhaps most important, the VSB is produced entirely by thin film deposition and optical lithography, and so can be fabricated in large format arrays. Also, the large noise margin of the SQUID amplifiers makes it possible to build multiplexers which can read out many bolometers through a single amplifier.

\section{Status of VSB Development}

Because of the attractive properties of the VSB, a number of groups are actively pursuing development. Much of the work in the open literature comes from the Berkeley group which will be featured here. More detailed descriptions of the Berkeley devices including illustrations can be found at http://bolo.berkeley.edu/. Leg-isolated bolometers with round (spiderweb) mesh absorbers have been successfully produced by thin film deposition and optical lithography [3]. As with conventional spiderweb bolometers, these devices will be used with undersampled horn-coupled arrays. Sources of excess noise have been explored and techniques to eliminate them have been found [4]. Excellent low frequency (1/f) noise performance has been demonstrated without ac bias, square format mesh absorber bolometers with folded legs suitable for compact arrays have been fabricated and tested [5]. Mechanical structures (legs and mesh absorbers) have been fabricated with high yield in a 1024 bolometer close-packed array format [5]. These arrays will be used to obtain Nyquist sampling for maximum mapping speed. A primitive hot electron (electron-phonon isolated) VSB has been successfully tested [1]. The Berkeley group (and others) are investigating antenna coupled VSB's. The antenna output can be coupled to the bolometer with a superconducting transmission line which can have very low loss at CMB frequencies and bolometer operating temperatures. The advantages of this approach are that the antennas can be polarization sensitive and that the antenna output can be split into a number of bands before detection by transmission line RF multiplexers and bandpass filters. This is particularly convenient for CMB polarization measurements where simultaneous measurements at several frequencies and two polarizations are required.

\section{Output Multiplexers}

Large format arrays of $10^{3}$ or more bolometers will be enabled by output multiplexing. Present technology requires one amplifier in the cryostat for each bolometer. Each amplifier requires $\sim 5$ wires. An array of $10^{3}$ bolometers would then require $5 \circ 10^{3}$ wires entering the cryostat. The technologies used to detect electromagnetic waves at infrared, optical and higher frequencies rely on output multiplexing schemes such as the CCD. The great scientific potential of the millimeter/submillimeter band will not be realized without an analogous output multiplexer scheme.

The group at the National Institute of Standards and Technology (NIST) was the first to address this critical need. The NIST group chose an approach in which SQUID's are used to switch the output of a row of detectors sequentially to a single amplifier [6]. The number of bolometers which can be read out through a single amplifier without increase in noise is given by the square of the ratio of the bolometer noise to the SQUID noise. The Berkeley group is exploring a frequency-domain multiplexer which ac biases each detector in a row with a different frequency and separates the signals with lock-in amplifiers at the SQUID output [7]. In this case, the limitation depends on the ratio of the slew rate of the SQUID to the flux noise [8]. A test chip has been fabricated with $\mathrm{Nb}$ thin-film lithography and signals from eight simulated bolometers have been successfully multiplexed and de-multiplexed with low crosstalk and noise. A design optimization has been completed and a more capable chip fabricated for tests with real bolometers. It is not clear at this stage whether time-domain or frequency-domain multiplexing will be best. Both appear to be capable of providing the output multiplexing that is absolutely essential for successful large format bolometer arrays.

\section{ACKNOWLEDGMENTS}

The authors are indebted to X. Meng for technical support in device fabrication. All devices were fabricated in the Berkeley Microfabrication Laboratory. 
This research was supported by the National Science Foundation, NASA, and by the Director, Office of Science, Office of Basic Energy Sciences of the U. S. Department of Energy under Contract No. DE-AC03$76 \mathrm{SF} 00098$. 


\section{REFERENCES}

1. Lee, A.T. et al., A Superconducting Bolometer With Strong Electrothermal Feedback, Appl. Phys. Lett. 69, 1801 (1996).

2. Lee, Shih-Fu et al., A Voltage-Biased Superconducting Transition Edge Bolometer with Strong Electro Thermal Feedback Operated at $370 \mathrm{mK}$, Applied Optics 37, 3391 (1998).

3. Gildemeister, J.M., Lee, A.T., and Richards, P.L., A Fully Lithographed Voltage-Biased Superconducting Spiderweb Bolometer, Appl. Phys. Lett. 74, 868 (1999).

4. Gildemeister, J.M., Lee, A.T., and Richards, P.L., A Model for Excess Noise in Voltage-Biased Superconducting Bolometers, Appl. Optics Lett. (in press).

5. Gildemeister, J.M., Lee, A.T., and Richards, P.L., Monolithic Arrays of Absorber-Coupled Voltage-Biased Superconducting Bolometers, Appl. Phys. Lett. 77, 4040 (2000).

6. Chervenak, J.A., et al., Superconducting Multiplexer for Arrays of Transition Edge Sensors, Appl. Phys. Lett. 74, 4043-4045 (1999).

7. Yoon, J., et al, Single Superconducting Quantum Interference Device Multiplexer for Arrays of Low-Temperature Sensors, Appl. Phys. Lett. 78, 371 (2001).

8. Yoon, J., et al, Single SQUID Multiplexer for Arrays of Voltage-Biased Superconducting Bolometers, presented at 9th International Workshop on Low Temperature Detectors, LTD-9, Madison Wisconsin, 23-37 July 2001. 